\title{
Very Short-Term Wind Power Forecasting Based on SVM-Markov
}

\author{
Shunhui Jiang ${ }^{1, \text { a }}$, Ruiming Fang ${ }^{1, b}$, Li Wang ${ }^{1, \mathrm{c}}$, Changqing Peng ${ }^{1, \mathrm{~d}}$ \\ ${ }^{1}$ College of Information Science and Engineering, Huaqiao University, Xiamen 361021, China

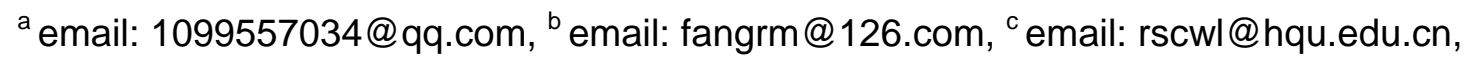 \\ demail: 344392094@qq.com
}

Keywords: Very short-term forecasting; SVM; Markov chain model; the confidence interval.

Abstract. Very short-term forecasting of wind power is important to scheduling staff's planning and wind turbine control. This paper has established a combined forecasting model based on Markov chain and support vector machine (SVM). Firstly, the SVM is used to model for wind power. Then, transition probability matrix is made based on Markov chain to modify for SVM prediction. Finally, the prediction confidence interval of combination forecasting model is given by method of fluctuation confidence interval. Verified by an example of a wind farm indicating that the combination forecasting model is better than a single SVM model on a variety of error indicators.

\section{Introduction}

In recent years, with the rapid growth of installed wind capacity, in order to ensure the grid's acceptance capacity for wind power, an accurate wind power forecasting is important. At present, the researches about the wind power forecasting models have obtained some results. These results include the single forecasting models and the combined forecasting models[1-3]. Although the combined models can get better forecasting accuracy, the error correction function is more important to the forecasting, because there are a lot of factors that effect the results of wind power forecasting and it's hard to accurately reflect the wind power fluctuation of wind farm no matter which forecasting algorithm is chosen. The Markov chain model has unique advantage in grasping the random fluctuating nature and has had a wide application field[4-5]. In this paper, the SVM regression model is used to forecast the very short-term wind power, and then the Markov chain model is used to correct the predicted wind power. In the meantime, considering the uncertainty of wind power forecasting[6-7], base on the research results about the uncertainty of wind power forecasting which have been made, this paper gives the confidence interval of the corrected prediction after an uncertainty analysis being made to it, providing more information for the dispatchers who need to make a reasonable dispatching and safety warning.

\section{The Model SVM-Markov}

\section{SVM Regression Model}

For a given known samples $\left(x_{1}, y_{1}\right),\left(x_{2}, y_{2}\right), \cdots,\left(x_{N}, y_{N}\right), x_{i} \in R^{n}, y_{i} \in R, i=1,2, \cdots, N, x_{i}$ is the input, $y_{i}$ is the output, SVM makes the input space of low-dimensional nonlinear mapping to the feature space of high-dimensional by using kernel functions, and carries on the linear regression in this space, getting the regression function in the following:

$$
f(x)=\sum_{i=1}^{N}\left(\overline{\alpha_{i}}-\overline{\alpha_{i}^{*}}\right) k\left(x, x_{i}\right)+\bar{b} .
$$

where $N$ is the number of samples, $\overline{\alpha_{i}}$ and $\overline{\alpha_{i}^{*}}$ is Lagrange multiplier, $x_{i}$ is input vector, $k\left(x, x_{i}\right)$ is kernel function, $\bar{b}$ is bias coefficient.

$$
\bar{b}=y_{i}-\sum_{i=1}^{N}\left(\overline{a_{j}^{*}}-\overline{a_{j}}\right) k\left(x, x_{i}\right)+\varepsilon .
$$

Due to the forecasting model of wind power is high degree of nonlinear model, and use the Gaussian function as the kernel function of prediction model, shown by the following: 


$$
k\left(x, x_{i}\right)=\exp \left(-\frac{\left\|x-x_{i}\right\|^{2}}{2 \sigma^{2}}\right)
$$

where $\sigma$ is the width of Gaussian kernel function.

In the forecasting model of SVM, penalty coefficient C and the width of kernel parameters $\sigma$ determines the performance of the forecasting model, so appropriate model parameters will make forecasting model to achieve better forecasting effect. In this paper, $(\mathrm{C}, \sigma)$ parameters are optimized by method of grid method and ten fold cross validation.

\section{Markov Chain Model}

The nature of the Markov chain is not influenced by the preceding time, which referring to future state of a system is only relevant to the current state. If a system has n states, the state of the previous time $t_{0}$ is $i$, and the state of the next time $t_{1}$ is $j$, the $p_{i j}$ is called one step transition probability. The set of the one step transition probability of makes up one step transition probability matrix $P^{(1)}=\left[p_{i j}\right]_{1 \leq i, j \leq n}$.

The $p_{i j}$ can be obtained by the formula: $p_{i j}=m_{i j} / m_{i}$, where $m_{i j}$ represents the number of times transferring from state $i$ to state $j \cdot m_{i}$ represents number of times the state $i$.

The forecasting model of Markov chain can be expressed as: $x_{k}=x_{0} P^{(1)}$, where $x_{0}$ is state probability vector of initial time, $x_{k}$ is state probability vector of next time.

\section{SVM-Markov Wind Power Forecasting Model and Uncertainty Analysis}

The specific steps of combined model are as follows:

(1)Setting up the SVM model to get the wind power prediction value. The residual error sequence is obtained by calculating the residual error between measured wind power and the predicted power.

(2)States division of residual error series. In order to minimize the average of the absolute value of the corrected wind power, this paper determines the number of states and size of intervals based on the thought of mean-mean square error.

(3)Establishing the Markov state transition probability matrix.

(4)Using the follow formula to correct the predicted power obtained from SVM model:

$y_{c}=y_{p} /(1+e)$.

where $y_{c}$ is the corrected wind power value, $y_{p}$ is the SVM prediction value, $e$ is the middle value of interval $i$. The paper selects the corrected wind power value which is closest to the current value as next time wind power value.

In this paper, we use the method of the fluctuation confidence interval to carry on the uncertainty analysis, giving the confidence level for the corrected values obtained by the SVM-Markov. fluctuation $p$ is defined as follows:

$$
p=100 \% \times\left(p_{i}-p_{i+1}\right) / p_{R} .
$$

where the $p_{i}$ and $p_{i+1}$ is the measured wind power at time $\mathrm{i}$ and $\mathrm{i}+1$ respectively, $p_{R}$ is the rated capacity of the wind turbine. The fluctuation confidence interval is defined as follows:

$P(X \leq R)=\lambda$.

where the $P$ represents probability, $X$ represents the absolute value of $p, \lambda$ indicates a given confidence level(confidence probability). Specific steps are as follows:

(1)Calculate the fluctuation $p$ according to formula (5), and then divide the fluctuation intervals.

(2)Divide the power intervals every $100 \mathrm{~kW}$.

(3)Establish power-fluctuation matrix.

(4)Select maximum fluctuation under the fluctuation confidence interval $R$, masked as $\Delta p_{\max , R}$.

(5)Calculate the range of power fluctuation. The upper and lower limits of the power fluctuation range are obtained by the following formula:

$$
\begin{aligned}
& p_{i l}=p_{i-1}-p_{R} \cdot \Delta p_{\max , R} \quad(i=2,3 \cdots, m-1) . \\
& p_{\text {iu }}=p_{i-1}+p_{R} \cdot \Delta p_{\max , R} .
\end{aligned}
$$


where $p_{i l}$ and $p_{i u}$ is lower limit and upper limit of power at sample i respectively, $p_{i-1}$ is measured power at sample i-1.

(6)Wind power correct. The power correction formula based on a certain confidence level $\lambda$ and fluctuation confidence interval $R$ is as follows:

$$
\overline{p_{i}}= \begin{cases}p_{\text {il }} & p_{i} \leq p_{\text {il }} \\ p_{i} & p_{\text {il }} \leq p_{i} \leq p_{\text {iи }} . \\ p_{\text {iи }} & p_{i} \geq p_{\text {iu }}\end{cases}
$$

where $\overline{p_{i}}$ is final corrected wind power value which contains confidence level, and the $p_{i}$ power prediction value which is obtained by the SVM-Markov.

\section{The Instance Analysis}

In order to verify the effectiveness of SVM-Markov model, we use real-time monitoring data of SCADA system from a wind turbine in Liaoning as simulation experiment. This wind turbine rated capacity is $1500 \mathrm{~kW}$. Data acquisition time is from 0:00 on March 16 to 24:00 on April 14, and the resolution is $1 \mathrm{~min}$, with 43200 set of historical data totally and each containing 78 data monitoring projects. This paper considers 18 projects only which have high correlation with the wind power, and takes wind power at $\mathrm{t}$ as the output of combined model, and takes all monitoring items at (t-1) as the inputs of combined model. Training and forecasting of models were carried out at Matlab2009a environment.

Firstly, this paper carried on 10min very short-term forecast of wind power based on SVM model so that it should mean the original data processing 10min. This paper selected data from 0:00 on March 24 to 20:00 on March 27, a total of $552 \times 19$ set of consecutive monitoring data. Data were normalized [8], and took the first $432 \times 19$ sets of data as the training sample of SVM model and after $120 \times 19$ set of data as a test sample of SVM model. This paper used a grid method and ten-fold cross-validation method for SVM parameters optimization, optimal parameter $C=16, \sigma=0.354$. The predictions comparison between SVM model and SVM-Markov model, as shown in Fig.1.

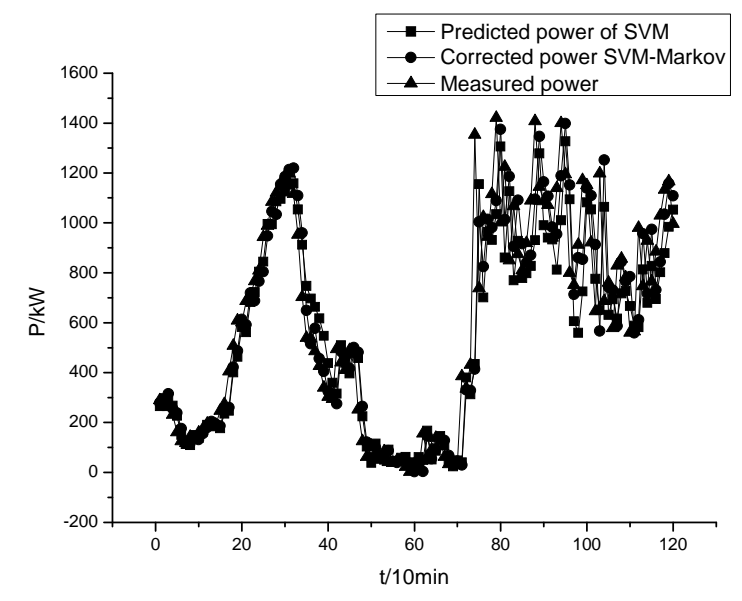

Fig.1 Predictions comparison

This paper used three error analysis indicators which were more commonly used [9], mean absolute error $E_{M A E}$, root mean square error $E_{R M S E}$, mean relative error $E_{M R E}$. Table 1 and Table 2 listed the error performance of each forecasting model and the different fluctuation confidence level. 
Table 1 Error performance of single and combination forecasting model

\begin{tabular}{cccc}
\hline & $E_{\text {MAE }}$ & $E_{\text {RMSE }}$ & $E_{\text {MRE }}$ \\
\hline SVM & 8.64 & 12.81 & 47.67 \\
SVM-Markov & 7.69 & 12.05 & 42.54 \\
\hline
\end{tabular}

Table 2 Error performance of different fluctuation confidence level

\begin{tabular}{cccc}
\hline & $E_{\text {MAE }}(\%)$ & $E_{\text {RMSE }}(\%)$ & $E_{\text {MRE }}(\%)$ \\
\hline$\lambda=95$ & 7.69 & 12.05 & 42.54 \\
$\lambda=90$ & 7.74 & 12.14 & 42.64 \\
$\lambda=85$ & 7.76 & 12.18 & 42.69 \\
$\lambda=80$ & 7.77 & 12.20 & 42.71 \\
$\lambda=75$ & 7.78 & 12.22 & 42.72 \\
$\lambda=70$ & 7.80 & 12.25 & 42.75 \\
\hline
\end{tabular}

From the results shown in Fig.1 and Table 1, the predicted results of SVM-Markov model established in this paper was closer to the measured value, and the mean absolute error was only $7.69 \%$, and it was lower than a single SVM prediction model which was $8.64 \%$. Table 2 showed that, the error performance of SVM-Markov model was slightly increased with the fluctuation of confidence level becoming smaller, which was due to fluctuations in the confidence level becoming smaller, the corresponding fluctuation confidence interval was also smaller, and individual predictions point was limited on the border with the forecast. When the fluctuation confidence interval was not the same size, which was the equivalent of changing the size of the forecast, and the prediction was smaller so that the error became large with the smaller fluctuations in the confidence interval.

\section{Conclusions}

This paper achieves wind power forecasting by using the combination model of the SVM and Markov, and get the following conclusions by verifying instance:

(1)Apply Markov model to correct the forecasting results of SVM. The state transition probability matrix makes the forecasting results to better fit the actual value by effectively correcting.

(2)The combination model of the SVM and Markov has higher forecasting accuracy than a single SVM model, the mean absolute error is reduced from $8.64 \%$ to $7.69 \%$, which verifying the effectiveness of proposed combination model.

(3)Using the method of the fluctuation confidence interval to analyze uncertainty of wind power forecasting, which is more meaningful than certainty result.

\section{Acknowledgements}

This work was financially supported by National Natural Science Foundation of China(51177039), Natural Science Foundation of Fujian Province(2012J01223), Major Scientific and Technological Innovation Project of Xiamen(3502Z2011008).

\section{References}

[1] Zhiyong Ding, Ping Yang, Xi Yang, et al. Automation of Electric Power Systems, 2012, 36(14): 131-135(in Chinese).

[2] Chenguang Niu, Cong Liu. Electric Power, 2011, 44(11): 73-77(in Chinese).

[3] Lin Ye, Peng Liu. Proceedings of the CSEE, 2011, 31(31): 102-108(in Chinese).

[4] Yinhua Huang, Jianchun Peng, Changchun Li, et al. Proceedings of the CSU-EPSA, 2011, 23(5): 131-136(in Chinese).

[5] Kani S A P, Ardehali M M. Energy Conversion and Management, 2011, 52(01): 738-745. 
[6] Songlin Zhou, Meiqin Mao, Jianhui Su. Proceedings of the CSEE, 2011, 31(25): 10-16(in Chinese).

[7] Jiannan Li, Ying Qiao, Zongxiang Lu, et al. Power System Protection and Control, 2010, 40(19): 7-13(in Chinese).

[8] Ying Liang, Ruiming Fang. Automation of Electric Power Systems, 2013, 37(14): 44-48(in Chinese).

[9] Man Xu, Ying Qiao, Zongxiang Lu. Automation of Electric Power Systems, 2011, 35(12): 20-26(in Chinese). 